\title{
PROOF OF ONE OPEN INEQUALITY OF LAUB-ILANI TYPE
}

\author{
LADISLAV MATEJÍČKA
}

Abstract. In this paper, we prove one new algebraic trigonometric inequality of Laub-Ilani type. This inequality was posted as the Conjecture 2.4 in the paper A. Y. Özban, "New AlgebraicTrigonometric Inequalities of Laub-Ilani type", Bull. Aust. Math. Soc., 2017, doi $10.1017 /$ S0004972717000156.

Mathematics subject classification (2010): 26D05, 26D07.

Keywords and phrases: Laub-Ilani inequalities, inequalities with power functions, algebraic-trigonometric inequalities.

\section{REFERENCES}

[1] ÖZBan, A. Y., New algebraic-trigonometric inequalities of laub-Ilani type, Bulletin of the Australian Mathematical Society, Volume 96, Issue 1, pp. 87-97, (2017), https://doi.org/10.1017/S0004972717000156.

[2] Cirtonje, V., On some inequalities with power-exponential functions, J. Inequal. Pure Appl. Math. 10 (2009), article ID 21. Google Scholar.

[3] Coronel, A. And Hunncas, F., Proof of three power-exponential inequalities, J. Inequal. Appl. 2014 (2014), article ID 509.CrossRef, Google Scholar.

[4] Laub, M., Problem E3116, Amer. Math. Monthly 92 (1985), 666. Google Scholar.

[5] Laub, M. And Ilani, I., A subtle inequality, Amer. Math. Monthly 97 (1990), 65-67. CrossRef, Google Scholar.

[6] Matejicka, L., Solution of one conjecture on inequalities with power-exponential functions, J. Inequal. Pure Appl. Math. 10 (2009), article ID 72. Google Scholar.

[7] Mitrinovic, D. S., Analytic inequalities, (Springer, Berlin, 1970). CrossRef, Google Scholar.

[8] MiYagi, M. AND NishizaWA, Y., A short proof of an open inequality with power-exponential functions, Aust. J. Math. Anal. Appl. 11 (2014), article 6. Google Scholar.

[9] Zeikit, A., Cirtoaje, V. and Berndt, W., Mathlinks forum, http://www .mathlinks.ro/Forum/viewtopic.php?t=118722, posted 11 Nov. 2006. Google Scholar. 